\title{
MMRS Coalitions: Continuing Their Efforts
}

\author{
David N. Gerstner, EMT-Paramedic; Brandon P. Sobera
}

$\mathrm{T}$ he Metropolitan Medical Response System (MMRS) was initially developed in 1996 in response to the Tokyo subway system sarin nerve agent gas attack and the Alfred P. Murrah Federal Building bombing in Oklahoma. Arguably the most important contribution of MMRS is coalition building. In many ways, MMRS could be viewed as the original health care coalitions.

As an example, Dayton (Ohio) MMRS (DMMRS) enhances and coordinates efforts to improve local and regional response capabilities for all forms of disasters: biological, manmade, or naturally occurring. DMMRS, like so many other MMRS cities, has responded to these challenges from its inception, and continues today preparing and implementing new response procedures that change the way responders react to different types of complex incidents.

DMMRS has helped develop multiple concepts including the Dayton MMRS Rescue Task Force for regional emergency medical services response to active shooter incidents, the adoption of SALT (sort, assess, lifesaving interventions, treatment/transport) triage, adopting the use of tourniquets, specialized response to Ebola and other special pathogens, and coordination of multidisciplinary response to radiological incidents. These concepts are being implemented to fill gaps in emergency response and were designed to enhance and coordinate local, regional, state, and intrastate response capabilities for highly populated areas that could be targeted by a terrorist attack using weapons of mass destruction. DMMRS used its coalition to develop a collaborative plan for a small number of agencies to respond under existing mutual aid agreements, and provide transports when required, both from the scene, and for interhospital transports for Ebola and other special pathogen patients. Finally, DMMRS has developed a regional plan for response to radiological emergencies with significant health consequences.

After its inception, MMRS became an "all-hazards" program. MMRS cities have spent years organizing resources to better manage large-scale incidents such as hazardous material accidents, epidemic disease outbreaks, and natural disasters that require specialized and carefully coordinated medical preparation and response, as well as enhancing preparations for terrorist threats.

From MMRS' beginnings under the Department of Health and Human Services and the 2001 acceptance of the contract to initiate Dayton MMRS, one goal has been kept in the forefront: to reduce human health consequences of disasters through planning, coalition creation, training, exercising, and equipping agencies for response to mass casualty incidents. Dayton MMRS and 123 other MMRS cities have long demonstrated that unifying regional partners increases the effectiveness of preparedness efforts by all involved. As people read about coalitions in this issue, they may want to look at the accomplishments and coalition-building in a nearby MMRS city.

\section{About the Authors}

MMRS Program, Dayton Fire Department, and Wright State University Boonshoft School of Medicine, Dayton, Ohio (Mr Gerstner); and MMRS Program, Dayton Fire Department, and Political Science and Criminal Justice Studies, University of Dayton, Dayton, Ohio (Mr Sobera).

Correspondence and reprint requests to David N. Gerstner, MMRS/ RMRS/EP Coordinator, Dayton Fire Department, 300 North Main Street, Dayton, OH 45402 (e-mail: david.gerstner@daytonohio.gov). 